\title{
Research on Testing System of Asynchronous Motor Based on LabVIEW
}

\author{
Renyin Song ${ }^{1, a}$ \\ ${ }^{1}$ Ningde Normal University, Ningde, Fujian, China, 352100 \\ ${ }^{a}$ email
}

Keywords: Asynchronous Motor, Testing System, LabVIEW

\begin{abstract}
Motor testing plays a very important role in the production of electrical machines. In recent years, the continuous development of computer technology has promoted the development of motor testing technology. According to the needs of asynchronous motor performance testing, the hardware and software designs of the testing system of asynchronous motor are completed on the LabVIEW software platform in this paper to provide some references for the related researchers.
\end{abstract}

\section{Introduction}

The continuous development of computer technology has injected new vitality into the traditional instruments. Traditional instruments are much more complex, having many test parameters. It is not easy to use and its limitations are slowly exposed. The motor is tested by virtual instrument test system composed of sensor, data acquisition card and industrial computer. The experimental data is entered into the computer through the sensor and data acquisition card, and the data is processed by the virtual experiment software. Data display, data management, data transmission, real-time detection and printing. Reduces the labor intensity of testing personnel, it is important to reduce human error, improve the test accuracy and repeatability and improve testing accuracy and testing speed, timely feedback to the motor design personnel for motor design parts adjusted to reach. The computer high speed data acquisition technology as the foundation, development can automatically collect motor rated parameters, static thrust, torque and other performance parameters of plugging, the back of multi-channel motor test system for the research and optimization of motor working process. It can evaluate the performance of the motor, improve production efficiency, reduce the work intensity and has important the significance of improving the quality of products.

The important component of the virtual instrument is the software development platform, which represents the entire drive software of the virtual instrument. Therefore, the level of the software development platform represents the level of the virtual instrument. With the rapid development of computer technology and software technology, a variety of special instrument development system is also becoming more and more powerful and perfect. The LabVIEW of the NI Company is a good represent. The graphical programming method adopts the idea of "what you see is what you get" to set up the man-machine interface. It provides a wealth of data collection, analysis and storage of the library function. LabVIEW provides a traditional program debugging tools, such as setting a breakpoint, single step, while providing a unique high brightness implementation tools, so that the process of debugging and development is more convenient. LabVIEW has a number of graphical operation templates for creating and running programs. These templates can be moved freely on the screen, and can be placed in any position as needed. Operation template is divided into the tools template, controls template and functions template. Tools templates provide basic tools for creating, modifying, and debugging programs. Controls templates include a variety of controls and indicators, 
mainly used to create the object in the front panel, constitute the program interface. Control templates can be added to the front panel input control and output display. Functions template contains the preparation of the code used in the process of a variety of functions and procedures.

\section{Hardware Design of Testing System of Asynchronous Motor Based on LabVIEW}

Asynchronous Motor. The rotor of an induction motor is placed in a rotating magnetic field, under the action of a rotating magnetic field, a rotating torque is obtained, and the rotor is rotated. The rotor is a rotating conductor, usually in a squirrel cage. Linear motor is a kind of mechanical energy, which can be directly converted into linear motion mechanical energy without any intermediate conversion mechanism. The linear motor drive device can simplify the structure of the whole device or system, which opens up a new field for the realization of high precision, fast response and high stability of the mechanical and electrical drive and control. Linear motor test is an important method to study linear motor. This test not only to test whether the products meet the design requirements of the technology, can also be found, prompted us to further examine the inherent law of master of linear motor, improve the level of theoretical research and engineering technology, it has an extremely important significance for the improvement of product design to improve the quality of motors. Servo driver has a matching driver software and industrial computer communication protocol, according to the needs of the servo motor control mode, set the software parameters, the servo motor for the corresponding thrust, speed and position control. When the test system for the detection of linear motor is in different speeds, the velocity in the servo drive motor loop control was used to drive the motor to complete the detection task.

Senor. Sensors play an important role in obtaining information and converting information, and sensor technology is closely related to information science, which is the general name of automatic detection and automatic conversion technology. A sensor is a device or device that can sense a prescribed measured and converted to an output signal according to a certain rule. The sensor to convert non electric power, it is a core part of the electrical measuring system. It will transform by non-electricity for the electricity, transport to the measurement circuit and indicating instrument or meter display. Sensor is a major part of realizing automatic detection and automatic control, sensor application, should be chosen according to the characteristics and performance index. The test data reflect the characteristics of the measured object, so the reliability of the sensor is of great significance. The reliability mainly includes the following two meanings: on the one hand can work normally under specified operating conditions; on the other hand refers to the performance of the sensor can be stable for a long time, the performance index does not change with time and environment change. So the reliability of the sensor should be given priority. The sensitivity is usually in the linear range of the sensors to the sensitivity of the sensor is good. When the measured changes, the corresponding output signal value change is relatively large, it is conducive to signal processing. But it should be noted that the high sensitivity of the sensor, and the external noise is not independent of the measurement is also easy to mix, it will be amplified system amplification, affect the accuracy of the measurement. Therefore, the sensor itself should have a high signal to noise ratio, as far as possible to reduce the interference signal from the outside world.

Data Acquisition Card. Data acquisition card is the analog signal collected by the sensor, through the data acquisition card into the digital signal can be processed by the data acquisition card, that is, analog/digital conversion, which is the data processing of the former Road work. In order to meet the needs of the computer and its compatible machines for data acquisition and control, many domestic and foreign manufacturers have produced a wide variety of data acquisition boards. This kind of standard bus technology board are a reference to the machine design and production, as long 
as the user send the card into the machine on the motherboard corresponding expansion slot, you can quickly and easily form a data acquisition and processing system, which saves hardware development time and investment, but also can make full use of soft the hardware resource of a computer, you can also use the users to concentrate on research, system design and programming on the theory and method of data acquisition and processing. Run time data and send to the machine, through the operation of the specific software to analyze these data, in order to determine the status of the current operating equipment, and then take the appropriate measures. The current commonly used data acquisition devices, in its system software design, more use of single task sequence mechanism.

\section{Software Design of Testing System of Asynchronous Motor Based on LabVIEW}

General Thinking. The software environment of the virtual instrument test system is developed by LabVIEW software. The main tasks are as follows: firstly, the user interface can be programmed quickly with the powerful graphical interface of LabVIEW. Users can simply operate the control test system on the main interface to complete the test task. Through the display control on the main interface, to test the waveform display, data display, etc., the preparation of the test function module, which is the core part of the software system. Including: initial parameter settings, test project selection, data collection, analysis and other modules; the test data for storage, display and analysis, and can callback data, reproduce test data and graphics. In determining the function and task division of the software system, can design the main program structure and program flow chart, a clear division of each module in the common data interface definition, each module of the program. This allows the program to be organized, easy to modify and maintain. The idea of structured and modular programming is adopted in the system. The structural design is required from top to bottom of the software from the local to the whole layers of segmentation, analysis of the various details of the mission, and the relationship between each other. The requirement of modular design process specific programming, according to the hierarchical structure of each individual task program module for the corresponding independent, according to the needs tasks call for each module. The virtual instrument software design is composed of the front panel and block diagram program. We can use the rich library functions of LabVIEW and powerful interface functions. They can be very convenient and quick to design the required procedures.

Module Design. According to the test content of linear motor performance test is divided into four main modules: the main interface, starting test, rated test, blocking test, and by the main interface call each test project. The routine test sequence is: start up test, rated test, blocking test. The test personnel can be carried out in accordance with the routine test order, can also be in the main interface to individually select one or more test items were tested, with a hint of each specific test items in the interface, and prompt content with the regular test sequence to the next item, to facilitate the test jump between projects. After the end of the test, we can carry out data callback. In each specific test items, according to different functions can be divided into the following modules. The data acquisition card initialization module is used to gather the test of different settings to control the amount of different acquisition card: the data acquisition module according to set parameters, such as test item list, number of sampling channel parameters measured parameters of motor the data processing module. According to the different test items written corresponding processing function, through the sampling value, it obtain the required parameters and generated by function fitting method, different curves calculated with the measurement coefficients set; storage module is used to store the test parameters and the curve obtained after the test in the file. 
Function Design. The main interface of the test software is to complete the motor test setup, test selection, data transfer and other operations to enter the corresponding test interface for testing. The main interface is composed of six function keys. Real time display area, fault alarm, curve display, test area, test data acquisition area control area and other control button data asynchronous motor test system based on LabVIEW control system. The software can completely replace the traditional operating platform to complete the testing process, and can be faster, more accurate adjustment variables and improve the detection efficiency of asynchronous motor sensor real-time updates. Interface of collected data, and the experimental process in the collected information to generate abnormal alarm, or even shut down, when the program started, the data is updated in real time. Each state has to start, stop and adjust the motor button that can make the operator in the running state. Testers can select one or more specific test items, if the error can be eliminated, new choice, which can be determined according to the selected project in order to enter the corresponding interface, test. The position of the test system is realized by controlling the rotary servo motor, and the position change of the rotary servo motor can be realized by the lead screw nut to drive the linear motor to run the corresponding displacement. The test real-time control system at present, the operator variable according to the test requirements of mediation test to display data in real-time, stable, click on the data acquisition button, the software will collect the data stored in the database. After completion of the test, the operator shut down the drive motor and the loading motor, according to the need of the test data in the database for retrieval and fitting performance curve is generated. Finally enter the settings interface settings, report parameters, generate reports and print pressure.

\section{Conclusion}

The asynchronous motor testing system based on LabVIEW ensures the effective implementation of the various parts. Compared with the previous testing system, its reliability is greatly improved. The advanced virtual instrument technology and data processing ability are applied in the testing system, improving the security of the system.

\section{Acknowledgements}

This research is the result of the "Dynamic Model Simulation of Three-phase Asynchronous Motor Based on LabVIEW” (Grant No. 2013F29).

\section{References}

[1] Chen Zheng, Wang Ying, Intelligent Robot, Vol. 12 (2015) No 7, p.68-71

[2] Li Han, Gong Lili, Shi Lijie, Zhou Yang, Zeng Xianguang, Railway Locomotive \& Car, Vol. 32 (2012) No 5, p.31-33

[3] Wang Xiulian, Guo Ruiguang, Bi Daqiang, Experimental Technology and Management, Vol. 32 (2015) No 12, p.73-78+83

[4] Zhao Ran, Wang Ying, Electronic Instrumentation Customers, Vol. 18 (2011) No 10, p.10-12 